\title{
Improved Electronic Voting Machine with Real Time Data Analysis
}

\author{
Anamika Sen \\ U.G. Student, Electronics and \\ Telecommunication \\ Engineering \\ Dwarkadas J. Sanghvi College \\ of Engineering, Mumbai \\ University
}

\author{
Malabika Sen \\ U. G. Student, Computer \\ Engineering \\ Dwarkadas J. Sanghvi College \\ of Engineering, Mumbai \\ University
}

\author{
Aarti Ambekar \\ Assistant Professor, Electronics \\ and Telecommunication \\ Engineering \\ Dwarkadas J. Sanghvi College \\ of Engineering, Mumbai \\ University
}

\begin{abstract}
Traditional manual voting system comes with shortcomings like additional cost of hiring personnels for the security of the storeboxes along with costs of papers, ink. The manual counting took upto four days to come up with proper results. Electronic Voting Machines help cut these costs, reducing the manual labor extensively, providing accurate results. This paper aims to do a real time analysis of the votes and ways in which data analysis can be used further.
\end{abstract}

\section{General Terms}

Microcontroller, Voting, Internet of Things

\section{Keywords}

Data Analysis, ESP8266, EVM, FRDM-KL25Z, ThingSpeak

\section{INTRODUCTION}

India is world's largest democracy and voting forms a crucial part in choosing the government. The conventional voting system in which the participants stamped across the candidate's name came with many flaws.[1] In an attempt to have a secured, faster and an error-free process, India adopted $\mathrm{e}$-Voting or Electronic Voting which were first used in General Elections, 1998. A simple EVM, for a small-scale purpose, can be made using a microcontroller and interfacing it with switches, or $\mathrm{m}^{*} \mathrm{n}$ matrix keyboard and an LCD to display the result. However, an EVM is not intended for use in political settings alone, but can be used by upcoming enterprises for polling too. In general, 'Electronic Voting' would consist of gathering of people's opinion, with the help of machinery which is computer supported to an extent.[2] Data is very much essential today as it helps understand the previous trends. Applying proper statistical analysis can help predict the demographic sentiments to a very large ex-tent. Using the results of an election, data analysis can be carried out which helps in finding out the current political scenario too. In this project, FRDM-KL25Z is used as the development platform. Touch sensor is used as the mode of input instead of the conventional method. WiFi module ESP8266 is used for sending the votes over the Internet and storing them in a database. Thingspeak, an open source Internet of Things (IoT) application and API is used to store and retrieve this data which can be then used for various Data Analysis applications, along with the real-time data analysis.

\section{LITERATURE REVIEW}

Similar works on designing Electronic Voting Machines using microcontrollers have been done before [2],[3],[4]. Microcontroller AT89C51 was used in[1] and atmega161 was used in [2]. A review of all the properties core in an EVM is explained in [1]. One problem which comes with the use of computerized application is the difficulty in using it. While many may find pressing switches, and following the operations to be simpler, a technologically-backward person finds it difficult. Thus, 'computer-disability' forms a serious issue, as it has been found that older part of the population face technical difficulties while performing these tasks.[1] The conventional systems had the results stored in the polling machine. With problems like technical faults and theft of the machine, the only measure left would be a Repeat poll. Hence, use of an RF module was suggested in [4] which would ensure storage of the poll results in a remote section and the EVM could be used only as the input device. In this paper, a similar approach is taken, replacing the transmit-ting module with WiFi module. As most of the ongoing work relates to voting in national elections, this thesis pro-poses different settings in which voting can be used and how the results can be further used effectively. Also, a touch sensor is used for taking in the votes/ polls, which is easier than switches, hence it eliminates the chances of error by less technically advanced people.

\section{BLOCK DIAGRAM}

There are 3 major blocks, which can be explained as follows :

\subsection{FRDM-KL25Z}

It is a low cost development platform built on ARM Cortex$\mathrm{M} 0+$ processor. It is supported by third-party development softwares too. mbed.org can be used to program this which provides online SDK. The capacitive touch sensor in this board is used as the mode of input.

\subsection{ESP8266}

This WiFi module is extremely cost-effective with integrated TCP/IP protocol stack that gives the micro-controller access to the WiFi network. In this project, it is used in sending the polls/ votes to the Thing-Speak platform.

\subsection{ThingSpeak API}

It is an IoT application and API to store and retrieve data from things from HTTP protocol over the Internet. It enables sensor logging applications. It has integrated MATLAB which enables analyzing and visualizing uploaded data. A channel needs to be created for the data to be logged in. The number of fields would be equal to the number of choices of votes we have. 


\section{4 mbed.org}

Additionally, developer.mbed.org was used for programming the microcontroller. It provides an online compiler for ARM based microcontrollers. Programming was done in C.

\section{METHODOLOGY}

The design proposed is a simple, low-cost and effective, which can be used for small to medium scale polling. It banks on the on-board capacitive touch sensor available on FRDMKL25Z, which is used as the mode of input. The touch sensor is divided into 2 parts, which serves as the two choices for the polls. It can be increased as needed. If the number increases to an extent which could not be supported by the area of the touchpad, external switches could be used. As the problems discussed in [4], there needs to be reliable storage of the polls/ votes, which leads to using the WiFi module. ESP-8266 is used in this regard, which is again a low-cost and effective $\mathrm{WiFi}$ module. The votes are thus sent over the Internet, to ThingSpeak API, which is used for the Real Time Data Analysis. The Serial Terminal is used here, instead of LCD for displaying the logging of votes.

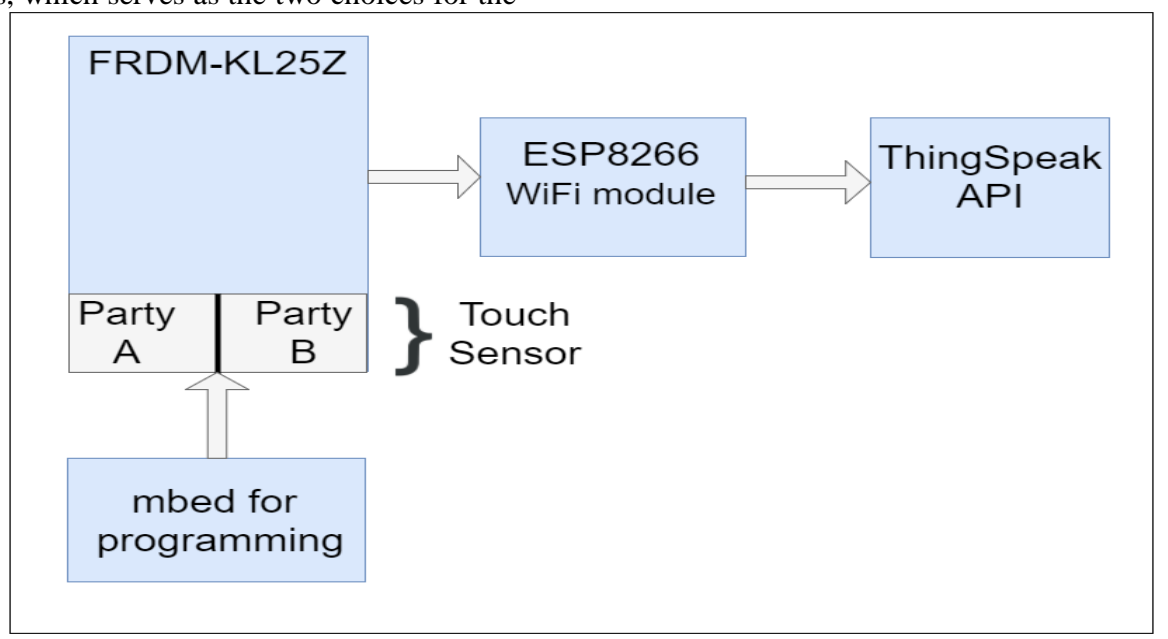

Fig 1: Block Diagram of the proposed project

\section{IMPLEMENTATION}

The Serial Terminal can be used to view the proper transmission of the votes/ polls.

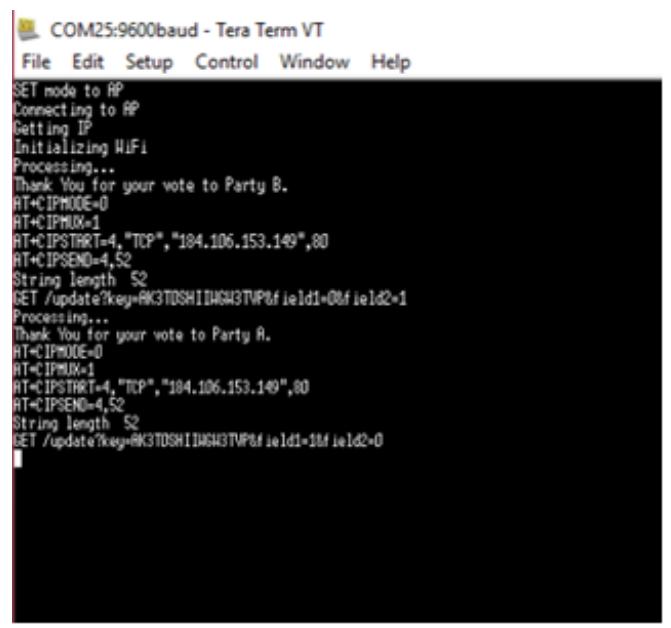

Fig 2: Wireless Transmission of votes on Serial Terminal Real Time Data analysis can be done using ThingSpeak API.

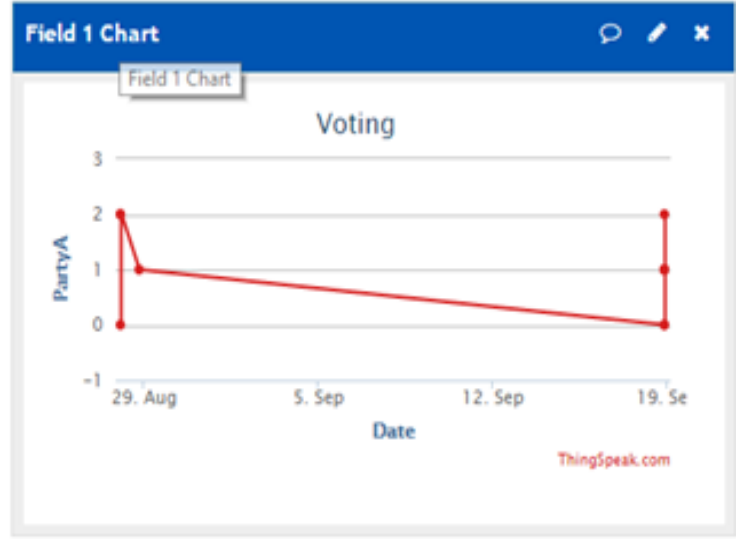

Fig 3: Votes/ Polls received by Party A over a period

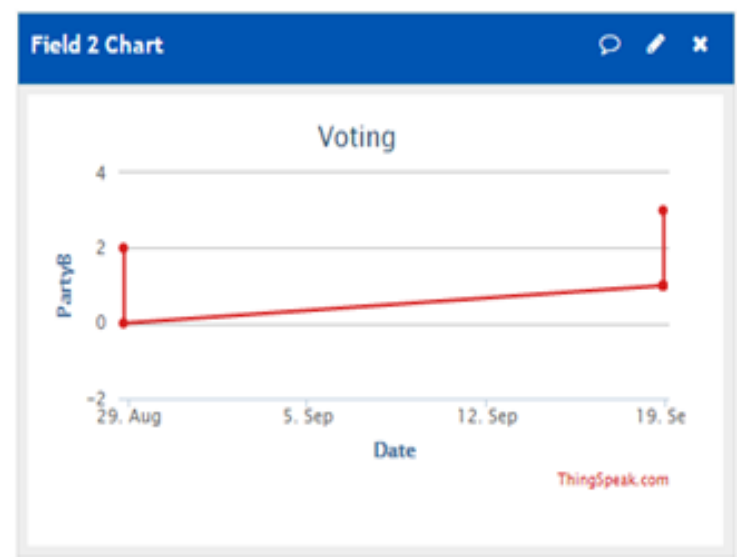

Fig 4: Votes/ Polls received by Party B over a period 
Further, using more APIs present, all the data added to the system can be viewed using the keys given while creating the channel.

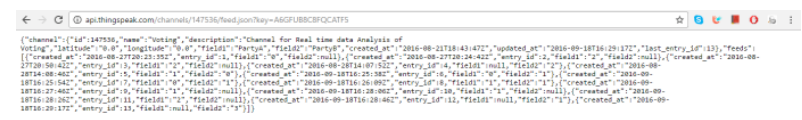

Fig 5: Log of all the Data added to the System

\section{APPLICATION AND FUTURE SCOPE}

This paper presents a view different than the conventional use of EVM. Since the data is stored in a remote database, the data is kept safe and secured. The different cases of application can be widely differentiated into the following.

\subsection{CONVENTIONAL USE}

If used traditionally, it can be used very well to predict an outcome of the election. Further, with the records of the previous elections, the changes in the current political scenario can be judged. Based on the region of the elections, and the winning party, community preferences can be identified. Regional, linguistic and a lot of other preferences can be examined too.

\subsection{NON-CONVENTIONAL USE}

New enterprises can use this in the survey of their new products. Polls can be taken from the target population. The data can further be analyzed according to different parameters, like the age of the population being surveyed, the region, the month of the poll etc. Many such factors come into picture with the introduction of data analysis. Comparing with data over the last few years, many factors could be found out which would help understand the previous and current market trends. Furthermore, Internet of Things could be applied here based on the data collected to perform any if-then scenarios.

\section{CONCLUSIONS}

Thus, this paper proposes a low-cost, non-polluting and energy-efficient way of polling. Using a WiFi module, the results are stored in a remote database as well as are available for analysis on a real-time basis. It can be used for fast-track voting, used in small-scale panchayat level, school level elections. Furthermore, using this setting, elections can be carried out in areas where electricity is not available. It is a very fine example of using environment-friendly resources when compared to the traditional election systems which used lakhs of ballot papers and ink. Since we employ the use of microcontroller, it becomes faster than the previous sys-tem. Additionally, it is not restricted just to the election aspect, but can be used effectively to find out various different demographic trends.

\section{ACKNOWLEDGEMENT}

We wish to thank Prof. Aarti G. Ambekar for her able and timely guidance in this paper. We would also like to thank Dwarkadas J. Sanghvi College of Engineering for providing us with the infrastructure.

\section{REFERENCES}

[1] D. Ashok Kumar, T. Ummal Sariba Begum, "Electronic Voting Machine - A Review", Pattern Recognition, Informatics and Medical Engineering (PRIME), 2012 International Conference

[2] Soumen Ghosh, "Development of Microcontroller Based Electronic Voting Machine (EVM)", IOSR Journal of Electrical and Electronics Engineering (IOSR-JEEE)

[3] Diponkar Paul and Sobuj Kumar Ray, "A Preview on Microcontroller Based Electronic Voting Machine," International Journal of Information and Electronics Engineering, Vol. 3, No. 2, March 2013

[4] Subhadeep Chakraborty, Dip Laha, Payel Kunda and Indrajeet Kunda, "Design of Secured Wireless EVM for Data Acquisition", International Journal of Advanced Research in Computer Science and Software Engineering, Vol. 5, Issue 9, September 2015

[5] M. A. Mazidi, J. G. Mazidi \& R. D. McKinlay, "The 8051 Microcontroller and Embedded Systems", Pearson Publication 2nd Edition, 2006

[6] C. Kenneth, J. Ayala \& D. V. Gadre,"The 8051 Microcontroller and Embedded System using Assembly and C", Cengage Learning, Edition 2010. 